\title{
Association between Physical Activity and Depressive Mood among Korean Adults with Chronic Diseases
}

\author{
Seon-Young Park', Kiheon Lee,*, Yoo Jin Um², Suekyoung Paek', In Sun Ryou' \\ 'Department of Family Medicine, Seoul National University Hospital, Seoul, Korea \\ ${ }^{2}$ Department of Family Medicine, Seoul National University Bundang Hospital, Seoul National University College of Medicine, Seongnam, Korea
}

\begin{abstract}
Background: This study aimed to investigate the association between physical activity and depressive mood among Korean adults with chronic diseases.

Methods: This study analyzed the 2014 Korean National Health and Nutrition Examination Survey data. The study was restricted to participants aged $\geq 20$ years who had completed the survey and were not diagnosed as having depression. After exclusion, 4,676 participants were included in the final analysis. Subjects were categorized according to their level of physical activity. Physical activity was assessed using the International Physical Activity Questionnaire. Patients were categorized into two groups according to depressive traits (normal and depressed) based on the 9-item Patient Health Questionnaire scores.

Results: In univariate analysis, significant associations were observed between the level of physical activity and depressive mood in both chronic and healthy groups. Participants with higher levels of physical activity had lower risks of depressive mood than those with lower levels of physical activity, especially patients with chronic diseases (odds ratio [OR], 0.47; 95\% confidence interval [CI], 0.31-0.73). Similar trends were observed in multivariate logistic regression analysis (OR, 0.54; 95\% CI, 0.34-0.88).

Conclusion: Physical activity significantly decreased the development of depressive mood among Korean adults with chronic diseases compared with their healthy counterparts. Physical activity must be emphasized in patients with chronic diseases.
\end{abstract}

Keywords: Exercise; Depression; Chronic Disease; Korean; Adult

Received: March 6, 2017, Revised: May 26, 2017, Accepted: July 11, 2017

${ }^{*}$ Corresponding Author: Kiheon Lee https://orcid.org/0000-0002-7139-2342

Tel: +82-31-787-7801, Fax: +82-31-787-4834, E-mail: keyhoney@gmail.com 


\section{INTRODUCTION}

Non-communicable diseases (NCDs), also known as chronic diseases, are major causes of morbidity and mortality worldwide. According to the 2014 cause-of-death statistics in Korea, $54.2 \%$ of Koreans died from cancer, cardiovascular diseases, diabetes mellitus (DM), and chronic respiratory diseases. ${ }^{1)}$ The prevalence of chronic diseases is likely to continue to increase and result in increased socioeconomic burden. Moreover, this burden will continue to increase due to an increasing elderly population., ${ }^{2,3)}$

According to the 2014 Korean National Health and Nutrition Examination Survey (KNHANES), $6.7 \%$ of Korean adults aged $\geq 20$ years have an underlying depressive disorder. Depression is one of the most common mental illnesses and a major public health concern worldwide. ${ }^{4}$ Furthermore, depression is often accompanied by chronic illnesses, and the burden of disease among chronic patients is known to increase with concomitant mental health disorders. ${ }^{5)}$

Physical inactivity is among the 10 leading risk factors for death worldwide and is a major risk factor for NCDs such as cardiovascular diseases, DM, and some cancers. ${ }^{6)}$ In addition, studies have discussed the relationship between physical activity and depression; it has been reported that physical inactivity increases the risk of depression, and physical activity has been reported to have a therapeutic effect on depression. ${ }^{7,8)}$

In patients with chronic diseases, the chronic disease itself may increase the prevalence of depression. Furthermore, it is thought that the lack of physical activity increases the risk of depression and adversely affects the course of the chronic disease due to the functional impairment and loss of productivity that accompany depression., ${ }^{9,10)}$

Therefore, physical activity is not only important in managing the chronic disease itself, but also in reducing the adverse effects of diseases when accompanied by depression. However, there is a lack of research on the relationship between physical activity and depression in certain disease groups. Although most studies have separately analyzed the relationships between physical activity and depression, depression and chronic disease, and physical activity and chronic disease, there insufficient studies linking these aspects.

The purpose of this study was to investigate the relationship between physical activity and depressive mood among Korean adults with chronic diseases. We hypothesized that the relationship between physical activity and depressive mood is more significant in people with chronic illnesses than in their healthy counterparts.

\section{METHODS}

\section{Study Population}

This study was conducted among Korean adults aged $\geq 20$ years who participated in the 2014 KNHANES. Of the 5,976 participants, 265 who were already diagnosed with depression were excluded. Within the limits of retrospective studies, it is best to exclude the deterioration of physical activity due to depression. Participants with missing data (e.g., no responses regarding biological, demographic, or socioeconomic status) were also excluded. In total, 4,676 participants were included in the present study (1,643 patients with chronic diseases and 3,033 in the control group) (Figure 1). Approval for the study was exempted by the Institutional Review Board of Seoul National University Bundang Hospital (IRB no., X-1703-388-901) because we analyzed publicly available data.

\section{Variables and Measurement}

\section{1) Chronic disease and depressive mood}

The present study included the following chronic diseases of interest: cancer, hypertension (HTN), DM, dyslipidemia, stroke, cardiovascular

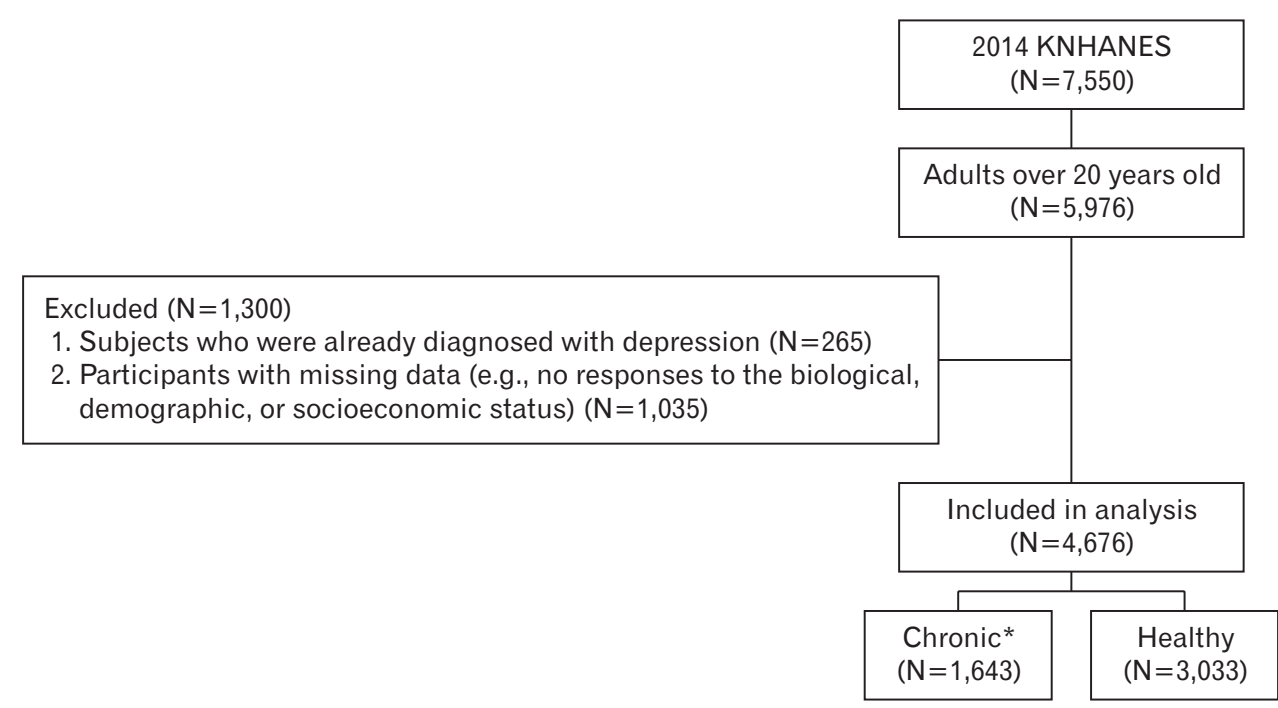

Figure 1. Flow chart of the subjects included in study. KNHANES, Korean National Health and Nutrition Examination Surveys. *Chronic diseases were limited to cancer, hypertension, diabetes, dyslipidemia, stroke, cardiovascular disease, and asthma. 
diseases, and asthma. Cardiovascular diseases included myocardial infarction and angina pectoris. Each chronic disease was diagnosed by a doctor. Depressive mood was assessed using the 9-item Patient Health Questionnaire (PHQ-9) scores and defined as a PHQ-9 score of $\geq 5$. ${ }^{11)}$ The PHQ-9 has been recommended for depression screening and has nine questions with scores ranging from 0 (not at all) to 3 (nearly every day) for each question (maximum score=27). ${ }^{12}$ )

\section{2) Physical activity}

We used the International Physical Activity Questionnaire-Short Form (IPAQ-SF) to assess physical activity level as it is considered a cost-effective tool. ${ }^{13)}$

The metabolic equivalent of task-minutes (MET-min) unit calculation used in the measurement of physical activity was based on the questionnaire items of the original data and IPAQ-SF. Walking, moderate activity (e.g., jogging, golf, pilates, or any other activity that causes a slight increase in breathing or heartbeat), and intense activity (e.g., running, swimming, mountain climbing, basketball, or any other activity that causes a substantial increase in breathing or heartbeat) were calculated as 3.3, 4.0, and 8.0 METs, respectively. ${ }^{14,15)}$ We calculated the instances of walking, moderate activity, and intense activity for at least 10 minutes each during the last week. The duration of each physical activity (days, hours, and minutes) was converted to minutes and summed up (MET-min=MET level $\times$ activity minutes $\times$ days).

A total value of less than 600 MET-min, between 600 and 2,999 METmin, and more than 3,000 MET-min were defined as low, moderate, and high, respectively. ${ }^{16)}$

\section{3) Biological, demographic, and socioeconomic variables}

Based on previous studies, ${ }^{8,17)}$ several variables affecting physical activity and depressive mood were assessed. ${ }^{18)}$ Educational level was classified into completed elementary school or less, completed middle school/high school diploma, and completed university or higher. Income level was classified by dividing the population group income data into three groups: lowest, middle, and highest tertile. Participants' marital status was classified as married with spouse, married but without spouse, and unmarried. Residential areas were classified into three categories: capital, metropolitan, and town/city. The degree of obesity was divided into $23.0,23.0-24.9$, and $25.0 \mathrm{~kg} / \mathrm{m}^{2}$, based on body mass index (BMI). Smoking status was classified into smokers, ex-smokers, and never-smokers. Alcohol consumption status was classified into never, drinking, and risky drinking. Risky drinking was defined as drinking 2 or more times a week and drinking seven or more standard drinks per day (five drinks for women). ${ }^{19,20)}$

\section{Statistical Analysis}

To determine the baseline characteristics according to physical activity level, numbers (\%) or mean values were calculated. Logistic regression analysis was used to determine the relationship between physical activity and depressive mood, according to the presence or absence of chronic diseases. Model A was adjusted for age and sex. Model B was adjusted for socioeconomic factors (education, residence, household income, spouse status), and model $\mathrm{C}$ was adjusted for health behavior factors (smoking, alcohol use, BMI) in addition to all variables in model A. Model D was adjusted for age, sex, socioeconomic factors, and health behavior factors. $\mathrm{P}<0.05$ was considered statistically significant. All statistical analyses were performed using Stata ver. 14.0 for Windows (Stata Corp., College Station, TX, USA). Because we only used data for 1 year, raw data were analyzed without additional weighting.

Table 1. General characteristics of the study population $(N=4,676)$

\begin{tabular}{|c|c|c|c|}
\hline Characteristic & $\begin{array}{l}\text { Healthy } \\
(n=3,033)\end{array}$ & $\begin{array}{l}\text { Chronic* }^{*} \\
(n=1,643)\end{array}$ & P-value \\
\hline Age (y) & $44.3 \pm 15.2$ & $62.8 \pm 12.9$ & $<0.001$ \\
\hline Sex & & & 0.949 \\
\hline Male & $1,297(42.8)$ & 701 (42.7) & \\
\hline Female & $1,736(57.2)$ & $942(57.3)$ & \\
\hline Education & & & $<0.001$ \\
\hline Elementary & $365(12.0)$ & $708(43.1)$ & \\
\hline Middle/high school & $1,400(46.2)$ & $654(39.9)$ & \\
\hline University & $1,266(41.8)$ & $279(17.0)$ & \\
\hline Household income & & & $<0.001$ \\
\hline Lowest & $331(10.9)$ & $506(30.9)$ & \\
\hline Middle & $1,734(57.3)$ & $804(49.1)$ & \\
\hline Highest & $961(31.8)$ & $327(20.0)$ & \\
\hline Residence & & & 0.032 \\
\hline Capital & $634(20.9)$ & $292(17.8)$ & \\
\hline Metropolitan & $821(27.1)$ & $451(27.4)$ & \\
\hline Town/city & $1,578(52.0)$ & $900(54.8)$ & \\
\hline Spouse status & & & $<0.001$ \\
\hline With spouse & $2,151(71.0)$ & $1,222(74.4)$ & \\
\hline Married but without spouse & $216(7.1)$ & $362(22.1)$ & \\
\hline Unmarried & $664(21.9)$ & $58(3.5)$ & \\
\hline Body mass index & & & $<0.001$ \\
\hline Under/normal & $1,575(51.9)$ & $563(34.3)$ & \\
\hline Overweight & $679(22.4)$ & $402(24.5)$ & \\
\hline Obesity & $778(25.7)$ & $677(41.2)$ & \\
\hline Smoking & & & $<0.001$ \\
\hline Never-smoker & $1,856(61.7)$ & $972(60.3)$ & \\
\hline Ex-smoker & $503(16.7)$ & $393(24.4)$ & \\
\hline Smoker & $649(21.6)$ & $246(15.3)$ & \\
\hline Alcohol use & & & $<0.001$ \\
\hline Never & $267(8.9)$ & $315(19.5)$ & \\
\hline Drinking & $2,380(79.1)$ & $1,164(72.0)$ & \\
\hline Risky drinking & $362(12.0)$ & $137(8.5)$ & \\
\hline Physical activity level & & & 0.011 \\
\hline Low & $1,287(42.4)$ & $772(47.0)$ & \\
\hline Moderate & $1,390(45.8)$ & $689(41.9)$ & \\
\hline High & $356(11.8)$ & $182(11.1)$ & \\
\hline 9-Item Patient Health Questionnaire & & & $<0.001$ \\
\hline Normal $(<5)$ & $2,442(80.5)$ & $1,279(77.8)$ & \\
\hline Depressed $(\geq 5)$ & 591 (19.5) & $364(22.2)$ & \\
\hline
\end{tabular}

Values are presented as mean \pm standard deviation or number (\%), unless otherwise stated.

${ }^{*}$ Chronic diseases were limited to cancer, hypertension, diabetes, dyslipidemia, stroke, cardiovascular disease, and asthma. ${ }^{\dagger}$ Calculated by one-way analysis of variance or chi-square analyses. 


\section{RESULTS}

\section{Baseline Characteristics of the Study Participants}

The general characteristics of the study population according to health status are shown in Table 1. Of the 4,676 participants, 1,643 were diagnosed with chronic disease, while 3,033 were without chronic disease. The mean age of the patients with chronic diseases was slightly higher.

Participants in the healthy group were more likely to be younger and more educated and to have a higher income and lower BMI. The healthy group included a higher proportion of unmarried participants, who were also more likely to be smokers and risky drinkers. The chronic disease group had a higher proportion of participants with low physical activity and depressive mood. However, there was no significant difference in the proportion of subjects belonging to either sex.

\section{Association between Physical Activity and Depressive Mood}

Table 2 shows the results of the logistic regression analysis of the risk of depressive mood according to physical activity levels. In univariate analysis, significant associations were observed between levels of physical activity and depressive mood in both chronic disease and healthy groups. Participants with higher levels of physical activity had lower risks of depressive mood than those with lower levels of physical activity (odds ratio [OR], 0.62; 95\% confidence interval [CI], 0.45-0.85), especially among chronic disease patients (OR, 0.47; 95\% CI, 0.310.73). Similar trends were observed in multivariate logistic regression analysis (chronic disease group: OR, 0.54; 95\% CI, 0.34-0.88; healthy group: OR, 0.73; 95\% CI, 0.59-0.89).

According to sex, the risk of depressive mood decreased as the degree of physical activity increased in women without chronic diseases (OR, 0.51; 95\% CI, 0.33-0.80); however, there was no significant difference in men before and after adjustment for covariates. In the chronic disease group, moderate levels of physical activity reduced the risk of depressive mood in both men and women compared with those with lower levels (men: OR, 0.57; 95\% CI, 0.37-0.88; women: OR, 0.56; 95\% CI, 0.41-0.77). As demonstrated earlier, the decrease in the risk of depressive mood according to physical activity was greater in patients with chronic illnesses. However, the dose-response association is not clear (Table 2).

\section{DISCUSSION}

This study analyzed the association between physical activity and depressive mood among Korean adults aged $\geq 20$ years who participated in the 2014 KNHANES. We found that the higher the amount of physical activity, the lower the risk of depressive mood. In particular, when differences according to the presence or absence of chronic diseases were compared, the decrease in the risk of depressive mood was greater among patients with chronic diseases. The reason for the greater association of physical activity in chronically ill individuals is thought to be that the psychological effect (mentioned below) is greater than the physiological effects of exercise. Regular exercise will increase aware-

Table 2. Odds ratios for the development of depressive mood according to physical activity level

\begin{tabular}{|c|c|c|c|c|c|c|}
\hline \multirow{3}{*}{ Variable } & \multicolumn{6}{|c|}{ Level of physical activity } \\
\hline & \multicolumn{3}{|c|}{ Healthy $(n=3,033)$} & \multicolumn{3}{|c|}{ Chronic $(n=1,643)$} \\
\hline & Low & Moderate & High & Low & Moderate & High \\
\hline \multicolumn{7}{|l|}{ Total } \\
\hline Unadjusted & 1 & $0.69(0.57-0.83)$ & $0.62(0.45-0.85)$ & 1 & $0.55(0.43-0.71)$ & $0.47(0.31-0.73)$ \\
\hline Model A & 1 & $0.70(0.58-0.85)$ & $0.67(0.49-0.92)$ & 1 & $0.57(0.44-0.74)$ & $0.54(0.35-0.84)$ \\
\hline Model B & 1 & $0.72(0.59-0.88)$ & $0.68(0.49-0.94)$ & 1 & $0.59(0.45-0.77)$ & $0.57(0.37-0.90)$ \\
\hline Model C & 1 & $0.71(0.58-0.86)$ & $0.72(0.52-0.99)$ & 1 & $0.58(0.45-0.76)$ & $0.53(0.33-0.84)$ \\
\hline Model D & 1 & $0.73(0.59-0.89)$ & $0.73(0.53-1.01)$ & 1 & $0.59(0.45-0.78)$ & $0.54(0.34-0.88)$ \\
\hline \multicolumn{7}{|l|}{ Men } \\
\hline Unadjusted & 1 & $0.77(0.55-1.09)$ & $0.96(0.61-1.52)$ & 1 & $0.57(0.37-0.89)$ & $0.50(0.26-0.95)$ \\
\hline Model A & 1 & $0.77(0.55-1.09)$ & $0.92(0.58-1.47)$ & 1 & $0.58(0.37-0.90)$ & $0.51(0.27-0.97)$ \\
\hline Model B & 1 & $0.78(0.55-1.11)$ & $0.95(0.59-1.50)$ & 1 & $0.58(0.37-0.93)$ & $0.53(0.27-1.02)$ \\
\hline Model C & 1 & $0.80(0.56-1.12)$ & $1.00(0.63-1.60)$ & 1 & $0.63(0.40-0.99)$ & $0.53(0.26-1.05)$ \\
\hline Model D & 1 & $0.80(0.56-1.14)$ & $1.00(0.63-1.62)$ & 1 & $0.61(0.38-0.99)$ & $0.52(0.26-1.05)$ \\
\hline \multicolumn{7}{|l|}{ Women } \\
\hline Unadjusted & 1 & $0.68(0.54-0.86)$ & $0.51(0.33-0.80)$ & 1 & $0.56(0.41-0.77)$ & $0.57(0.32-1.05)$ \\
\hline Model A & 1 & $0.67(0.53-0.85)$ & $0.50(0.32-0.79)$ & 1 & $0.56(0.41-0.77)$ & $0.57(0.32-1.05)$ \\
\hline Model B & 1 & $0.69(0.54-0.88)$ & $0.51(0.32-0.81)$ & 1 & $0.59(0.43-0.82)$ & $0.63(0.34-1.18)$ \\
\hline Model C & 1 & $0.67(0.53-0.85)$ & $0.53(0.34-0.84)$ & 1 & $0.56(0.41-0.78)$ & $0.54(0.29-1.02)$ \\
\hline Model D & 1 & $0.68(0.53-0.87)$ & $0.54(0.34-0.86)$ & 1 & $0.59(0.42-0.82)$ & $0.60(0.32-1.14)$ \\
\hline
\end{tabular}

Values are presented as odds ratio (95\% confidence interval). Model A: adjusted for age and sex; model B: adjusted for age, sex, and socioeconomic factors (education, residence, household income, and spouse status); model C: adjusted for age, sex, and health behavior factors (smoking, alcohol use, and BMI); and model D: adjusted for age, sex, socioeconomic factors (education, residence, household income, and spouse status), and health behavior factors (smoking, alcohol use, and BMI). Statistically significant results are written in bold $(P<0.05)$.

BMl, body mass index. 
ness of self-efficacy or confidence in the body, and the self-esteem resulting from regular exercise is more important in those with chronic disease than in healthy people. ${ }^{9)}$

A previous study have reported a tendency for a dose-response association; ${ }^{17)}$ we propose that the lack of clarity of the dose-response in our study was because of the fact that we separated the degree of physical activity into only three groups.

Physical activity, the main focus of this study, is widely known to reduce the risk of coronary heart disease, stroke, DM, and other chronic diseases. ${ }^{21-23)}$ While studies have described the association between physical activity and depression, the mechanism behind this association remains unclear. Exercise has been shown to alter norepinephrine activity, decrease hypothalamic-pituitary-adrenal axis activity, and increase beta-endorphin secretion, resulting in improved mood. ${ }^{24)}$ In addition to these physiological responses, psychological mechanisms are also known to be involved. These factors might explain why the OR for depressive mood was significant for women, but not for men, in the context of high levels of physical activity in our study. ${ }^{25)}$

With regard to the role of depression in increasing the burden of disease among patients with chronic diseases, it can be inferred from our findings that physical activity reduces the risk of depressive mood and influences disease outcomes. ${ }^{10)}$ In addition, a previous study found that the level of physical activity was low among Korean adults with chronic diseases such as HTN and DM. ${ }^{26)}$ Therefore, the importance of physical activity among patients with chronic illnesses should be strongly emphasized.

The study limitations are as follows. First, because this was a crosssectional study, it was difficult to determine the causal relationship between depressive mood and physical activity. Second, there is a possibility of recall bias as the classification of depressive mood and physical activity was based on a retrospective self-report questionnaire. Third, we identified an association between depressive mood and physical activity in chronic illnesses, but we have not yet identified any differences in disease outcomes due to decreased depressive symptoms. Fourth, although the KNHANES data are representative, we only analyzed data from 2014, when the PHQ-9 was newly introduced. In addition, because only the amount of leisure-time physical activity was included, the correlation with occupational physical activity was not reflected. Further validation is required through prospective studies involving a larger sample size to substantiate the recommendation of increased physical activity among people with chronic diseases.

In conclusion, higher levels of physical activity significantly decreased the development of depressive mood among Korean adults with chronic diseases, compared to their healthy counterparts. Thus, by recommending appropriate physical activity in patients with chronic illnesses, we can reduce the risk of depressive mood and expect an improvement in the course of the disease, although follow-up studies are necessary to evaluate chronic disease outcomes. Overall, the importance of physical activity must be emphasized in chronic disease patients.

\section{CONFLICT OF INTEREST}

No potential conflict of interest relevant to this article was reported.

\section{REFERENCES}

1. Korean Statistical Information Service. Cause-of-death statistics in Korea, 2014 [Internet]. Daejeon: Statistics Korea; 2014 [cited 2017 Feb 15]. Available from: http://kosis.kr/ups/ups_01List.jsp.

2. Institute for Health Metrics and Evaluation. GBD country profiles, 2013 [Internet]. Seattle (WA): Institute for Health Metrics and Evaluation; 2013 [cited 2017 Feb 15]. Available from: http://www.healthdata. org/results/country-profiles.

3. Centers for Disease Control and Prevention, Kim YA, Oh KY. Prevalence of chronic diseases by household income among adults in Korea, 2013. Public Health Wkly Rep 2015;8:33-6.

4. Moussavi S, Chatterji S, Verdes E, Tandon A, Patel V, Ustun B. Depression, chronic diseases, and decrements in health: results from the World Health Surveys. Lancet 2007;370:851-8.

5. Katon WJ, Lin EH, von Korff M, Ciechanowski P, Ludman EJ, Young B, et al. Collaborative care for patients with depression and chronic illnesses. N Engl J Med 2010;363:2611-20.

6. World Health Organization. Physical activity: fact sheet [Internet]. Geneva: World Health Organization; 2015 [cited 2017 Feb 15]. Available from: http://www.who.int/mediacentre/factsheets/fs385/en/.

7. Mikkelsen SS, Tolstrup JS, Flachs EM, Mortensen EL, Schnohr P, Flensborg-Madsen T. A cohort study of leisure time physical activity and depression. Prev Med 2010;51:471-5.

8. Dinas PC, Koutedakis Y, Flouris AD. Effects of exercise and physical activity on depression. Ir J Med Sci 2011;180:319-25.

9. Chapman DP, Perry GS, Strine TW. The vital link between chronic disease and depressive disorders. Prev Chronic Dis 2005;2:A14.

10. Egede LE. Major depression in individuals with chronic medical disorders: prevalence, correlates and association with health resource utilization, lost productivity and functional disability. Gen Hosp Psychiatry 2007;29:409-16.

11. Kroenke K, Spitzer RL, Williams JB. The PHQ-9: validity of a brief depression severity measure. J Gen Intern Med 2001;16:606-13.

12. Arroll B, Goodyear-Smith F, Crengle S, Gunn J, Kerse N, Fishman T, et al. Validation of PHQ-2 and PHQ-9 to screen for major depression in the primary care population. Ann Fam Med 2010;8:348-53.

13. Lee PH, Macfarlane DJ, Lam TH, Stewart SM. Validity of the International Physical Activity Questionnaire Short Form (IPAQ-SF): a systematic review. Int J Behav Nutr Phys Act 2011;8:115.

14. Craig CL, Marshall AL, Sjostrom M, Bauman AE, Booth ML, Ainsworth $\mathrm{BE}$, et al. International physical activity questionnaire: 12-country reliability and validity. Med Sci Sports Exerc 2003;35:1381-95.

15. International Physical Activity Questionnaire. IPAQ scoring protocol [Internet]. [place unknown]: International Physical Activity Questionnaire Group [cited 2017 Feb 15]. Available from: https://sites.google. $\mathrm{com} /$ site/theipaq/scoring-protocol.

16. Kim DH, Lee EJ, Lee JY, Lee DC. The association and the characteristics of the smoking status and differences in physical activity level in Korean adults: the sixth Korea National Health and Nutrition Exami- 
nation Survey (KNHANES VI-1), 2013. Korean J Fam Pract 2015;5(3 Suppl 2):510-6.

17. Camacho TC, Roberts RE, Lazarus NB, Kaplan GA, Cohen RD. Physical activity and depression: evidence from the Alameda County Study. Am J Epidemiol 1991;134:220-31.

18. Bauman AE, Reis RS, Sallis JF, Wells JC, Loos RJ, Martin BW, et al. Correlates of physical activity: why are some people physically active and others not? Lancet 2012;380:258-71.

19. Lee HK, Lee BH. The epidemiology of alcohol use disorders. J Korean Diabetes 2012;13:69-75.

20. Korea Centers for Disease Control and Prevention. The 5th Korea National Health and Nutrition Examination Survey (KNHANES V-1). Cheongju: Korea Centers for Disease Control and Prevention; 2010.

21. Huai P, Xun H, Reilly KH, Wang Y, Ma W, Xi B. Physical activity and risk of hypertension: a meta-analysis of prospective cohort studies. Hypertension 2013;62:1021-6.
22. Lambert EV, Bull F. Public health recommendations for physical activity in the prevention of type 2 diabetes mellitus. Med Sport Sci 2014;60:130-40.

23. Wen CP, Wai JP, Tsai MK, Yang YC, Cheng TY, Lee MC, et al. Minimum amount of physical activity for reduced mortality and extended life expectancy: a prospective cohort study. Lancet 2011;378:1244-53.

24. Blumenthal JA, Babyak MA, Moore KA, Craighead WE, Herman S, Khatri P, et al. Effects of exercise training on older patients with major depression. Arch Intern Med 1999;159:2349-56.

25. Teychenne M, Ball K, Salmon J. Physical activity and likelihood of depression in adults: a review. Prev Med 2008;46:397-411.

26. Jin HS, An AR, Choi HC, Lee SH, Shin DH, Oh SM, et al. Physical activity level of Korean adults with chronic diseases: the Korean National Health and Nutritional Examination Survey, 2010-2012. Korean J Fam Med 2015;36:266-72. 